\title{
Mesh reduction strategy: special element for modelling anisotropic materials with defects
}

\author{
Changyong $\mathrm{Cao}^{1}$, Aibing $\mathrm{Yu}^{2}$ \& Qing H. Qin ${ }^{1,3}$ \\ ${ }^{1}$ Research School of Engineering, \\ Australian National University, Australia \\ ${ }^{2}$ School of Materials Science and Engineering, \\ University of New South Wales, Australia \\ ${ }^{3}$ Department of Engineering Mechanics, \\ Henan University of Technology, China
}

\begin{abstract}
In this paper we present a hybrid finite element method (HFS-FEM) to model efficiently and accurately anisotropic materials with defects by developing special elements for elliptic hole/crack based on their associated Green's functions. The hybrid method is formulated based on two independent assumptions: intra-element field in terms of the combination of fundamental solutions and inter-element frame fields along the element boundary. A modified functional, which is satisfying the governing equation, boundary and continuity conditions between elements, is proposed to derive the element stiffness. In this work, the foundational solutions of the anisotropic materials following Stroh formalism are employed to approximate the intra-element displacement field of general elements, while the special fundamental solutions satisfying the required boundary conditions for hole or crack are used for the special elements containing defects. Two examples are presented to assess the performance of the proposed method. Numerical results obtained for the stress concentration factor (SCF) and stress intensity factor (SIF) are extremely accurate for the investigated cases.

Keywords: anisotropic elasticity, Green's function, hybrid finite element, Stroh formalism, stress intensity factor.
\end{abstract}




\section{Introduction}

In practice many structure are designed to involve holes for special purpose and in general there is no perfect materials existed. These facts make the stress analysis for materials with defects such as holes, cracks and inclusions is essential and important [1-5]. The mechanical behaviour of composite materials is usually studied by using anisotropic elasticity due to its anisotropic property. In literature, there are two main approaches dealing with the two-dimensional linear anisotropic elasticity. One is Lekhnitskii formalism [6] which begins with the stresses, the other is Stroh formalism [7, 8] which starts with the displacements, both of which are formulated by complex variable functions. Because of the limitations of the analytical solutions which are only available for problems with very simple geometry and boundary conditions [4, 9], numerical methods such as the finite element method (FEM), boundary element method (BEM) and mesh free method (MFM) are usually resorted to solve problems with multiple holes, cracks or inclusions under complicated boundary constraints and loading conditions $[9,10]$.

However, the mesh refinement near a hole or crack is required for FEM to achieve necessary accuracy, which is a very time-consuming and complex task [11]. To avoid the mesh refinement, a Green's function for two-dimensional linear anisotropic elasticity has been derived [7] and linear boundary elements were developed and extended to the problems of multi-holes, cracks and inclusions by utilizing special Green's functions [4, 12]. However, it is not appropriate to apply this special element to the problems whose holes are subjected to loads because of the traction-free-hole boundary condition. Recently a hybrid finite element formulation based on the fundamental solutions, called the HFS-FEM, was developed for solving three-dimensional elastic [13], thermal [14] and piezoelectric problems [15], which inherits the advantages of the Hybrid Trefftz FEM (HT-FEM) over the FEM and the BEM, such as the possibility of high accuracy using coarse meshes of high-degree elements, enhanced insensitivity to mesh distortion, great liberty in element shape, accurately representing various local effects without troublesome mesh adjustment $[10,16]$. Compared to the HT-FEM, HFS-FEM has simpler interpolation kernel expressions for intra-element fields (fundamental solutions) and avoids the coordinate transformation procedure required in the HT-FEM to keep the matrix inversion stable.

In this paper a new efficient and accurate HFS-FEM with special elements for elliptic hole/crack is developed based on the associated fundamental solutions to model the anisotropic materials with defects. The hybrid finite element method is formulated based on two independent displacements, one is the interior displacement approximations in terms of the combination of fundamental solutions, and another is the element frame displacement fields defined along the element boundary. The element boundary integral functional is used to enforce the assumed two fields and generate the final element stiffness equation. In this work, the foundational solutions of the anisotropic materials are employed to approximate the intra-element displacement field of general elements, while the 
special fundamental solutions satisfying the required boundary conditions for hole/crack are used for the special elements involving defects. Two examples are presented to demonstrate the accuracy and efficiency of the proposed method.

\section{Linear anisotropic elasticity}

\subsection{Basic equations and Stroh formalism}

In the Cartesian coordinate system $\left(x_{1}, x_{2}, x_{3}\right)$, if we neglect the body force $b_{i}$, equilibrium equations, stress-strain laws and strain-displacement equations for anisotropic elasticity can be written as [7]:

$$
\begin{gathered}
\sigma_{i j, j}=0 \\
\sigma_{i j}=C_{i j k l} e_{k l} \\
e_{i j}=\left(u_{i, j}+u_{j, i}\right) / 2
\end{gathered}
$$

where $i, j=1,2,3, \sigma_{i j}$ is the stress tensor, $e_{k l}$ the strain tensor, $C_{i j k l}$ the fourthrank anisotropic elasticity tensor, and $u_{i}$ the displacement vector. For convenience, matrices are represented by bold face letters in this paper and a comma followed by an index implies differentiation with respect to that index. The summation convention is invoked over repeated indices. The equilibrium equations can be rewritten in terms of displacements by substituting Eqs. (2) and (3) into Eq. (1),

$$
C_{i j k l} u_{k, j l}=0
$$

Eqs. (2)-(4) are completed by adding following boundary conditions:

$$
\begin{gathered}
u_{i}=\bar{u}_{i} \quad \text { on } \Gamma_{\mathrm{u}} \\
t_{i}=\sigma_{i j} n_{j}=\bar{t}_{i} \quad \text { on } \Gamma_{\mathrm{t}}
\end{gathered}
$$

where $\bar{u}_{i}$ and $\bar{t}_{i}$ are the prescribed boundary displacement and traction vector, respectively, $n_{i}$ is the unit outward normal to the boundary, and $\Gamma=\Gamma_{u}+\Gamma_{t}$ is the boundary of the solution domain $\Omega$.

For the generalized two-dimensional deformation of anisotropic elasticity $u_{i}$ can be assumed to depend on $x_{1}$ and $x_{2}$ only. Based on this assumption, the general solution to (4) can be written as $[8,17]$ :

$$
\mathbf{u}=2 \operatorname{Re}\{\mathbf{A f}(z)\}, \boldsymbol{\phi}=2 \operatorname{Re}\{\mathbf{B f}(z)\}
$$

where $\mathbf{u}=\left(u_{1}, u_{2}, u_{3}\right)^{T}$ is the displacement vector, $\boldsymbol{\phi}=\left(\varphi_{1}, \varphi_{2}, \varphi_{3}\right)^{T}$ is the stress function vector, $\operatorname{Re}$ stands for the real part of a complex number, $\mathbf{f}(z)=\left[f_{1}\left(z_{1}\right), f_{2}\left(z_{2}\right), f_{3}\left(z_{3}\right)\right]^{T}$ is a function vector composed of three holomorphic complex function $f_{\alpha}\left(z_{\alpha}\right), \alpha=1,2,3$, which is an arbitrary function 
with argument $z_{\alpha}=x_{1}+p_{\alpha} x_{2}$ and will be determined by satisfying the boundary conditions of the problems. $p_{\alpha}$ are the material eigenvalues with positive imaginary part, $\mathbf{A}=\left[\mathbf{a}_{1}, \mathbf{a}_{2}, \mathbf{a}_{3}\right]$ and $\mathbf{B}=\left[\mathbf{b}_{1}, \mathbf{b}_{2}, \mathbf{b}_{3}\right]$ are $3 \times 3$ complex matrices composed by the material eigenvector matrix associated with $p_{\alpha}$, which can be obtained by the following eigen relations [7]:

$$
\mathbf{N \xi}=p \boldsymbol{\xi}
$$

where $\mathbf{N}$ is a $6 \times 6$ foundational elasticity matrix and $\xi$ is a $6 \times 1$ column vector defined by

$$
\mathbf{N}=\left[\begin{array}{ll}
\mathbf{N}_{1} & \mathbf{N}_{2} \\
\mathbf{N}_{3} & \mathbf{N}_{1}^{\mathrm{T}}
\end{array}\right], \boldsymbol{\xi}=\left\{\begin{array}{l}
\mathbf{a} \\
\mathbf{b}
\end{array}\right\}
$$

where $\mathbf{N}_{1}=-\mathbf{T}^{-1} \mathbf{R}^{\mathbf{T}}, \mathbf{N}_{2}=\mathbf{T}^{-1}, \mathbf{N}_{3}=\mathbf{R} \mathbf{T}^{-1} \mathbf{R}^{\mathbf{T}}-\mathbf{Q}$ and the matrixes $\mathbf{Q}, \mathbf{R}$ and $\mathbf{T}$ are $3 \times 3$ matrices extracted from $C_{i j k l}$ as follows

$$
Q_{i k}=C_{i 1 k 1}, R_{i k}=C_{i 1 k 2}, T_{i k}=C_{i 2 k 2}
$$

The stresses can be obtained from the derivative of stress functions $\boldsymbol{\phi}$ as follows

$$
\left\{\sigma_{i 1}\right\}=2 \operatorname{Re}\left\{\mathbf{L} \mathbf{f}^{\prime}(z)\right\},\left\{\sigma_{i 2}\right\}=2 \operatorname{Re}\left\{\mathbf{B} \mathbf{f}^{\prime}(z)\right\}
$$

where

$$
L=\left[-p_{1} \mathbf{b}_{1},-p_{2} \mathbf{b}_{2},-p_{3} \mathbf{b}_{3},-p_{4} \mathbf{b}_{4}\right]
$$

\subsection{Foundational solutions}

\section{Fundamental solutions for general elements}

Consider an infinite homogeneous anisotropic elastic medium loaded by a concentrated point force (or line force for two-dimensional problems) $\hat{\mathbf{p}}=\left(\hat{p}_{1}, \hat{p}_{2}, \hat{p}_{3}\right)$ applied at an internal point $\hat{\mathbf{x}}=\left(\hat{x}_{1}, \hat{x}_{2}\right)$ far from the boundary. The Green's function of this problem has been found to be $[4,7]$

$$
\begin{aligned}
& \mathbf{u}=\frac{1}{\pi} \operatorname{Im}\left\{\mathbf{A}\left\langle\ln \left(z_{\alpha}-\hat{z}_{\alpha}\right)\right\rangle \mathbf{A}^{\mathbf{T}}\right\} \hat{\mathbf{p}} \\
& \phi=\frac{1}{\pi} \operatorname{Im}\left\{\mathbf{B}\left\langle\ln \left(z_{\alpha}-\hat{z}_{\alpha}\right)\right\rangle \mathbf{A}^{\mathbf{T}}\right\} \hat{\mathbf{p}}
\end{aligned}
$$

The corresponding stress components can be obtained by

$$
\begin{aligned}
& \sigma_{i 1}^{*}=-\phi_{, 2}=-\frac{1}{\pi} \operatorname{Im}\left\{\mathbf{B}\left\langle p_{\alpha} /\left(z_{\alpha}-\hat{z}_{\alpha}\right)\right\rangle \mathbf{A}^{\mathbf{T}}\right\} \hat{\mathbf{p}} \\
& \sigma_{i 2}^{*}=\phi_{, 1}=\frac{1}{\pi} \operatorname{Im}\left\{\mathbf{B}\left\langle 1 /\left(z_{\alpha}-\hat{z}_{\alpha}\right)\right\rangle \mathbf{A}^{\mathbf{T}}\right\} \hat{\mathbf{p}}
\end{aligned}
$$

\section{Fundamental solutions for special elements}

Consider an infinite anisotropic plate containing a traction-free elliptic hole under a concentrated force $\hat{\mathbf{p}}=\left(\hat{p}_{1}, \hat{p}_{2}, \hat{p}_{3}\right)$ applied at point $\hat{\mathbf{x}}=\left(\hat{x}_{1}, \hat{x}_{2}\right)$, as shown in Figure 1. The Green's function of this problem can be obtained by employing 


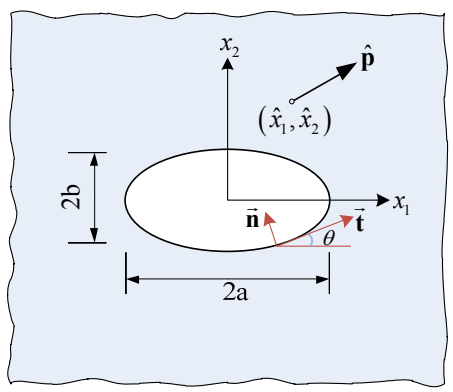

Figure 1: $\quad$ Schematic of the infinite anisotropic plate with an elliptical hole.

the conformal mapping technique to map the elliptical hole into a unit circle. The expressions of the Green function has been derived to be [4, 7]

$$
\begin{aligned}
& \mathbf{u}=\frac{1}{\pi} \operatorname{Im}\left\{\mathbf{A}\left\langle\ln \left(\varsigma_{\alpha}-\hat{\varsigma}_{\alpha}\right)\right\rangle \mathbf{A}^{\mathbf{T}}+\sum_{k=1}^{3} \mathbf{A}\left\langle\ln \left(\varsigma_{\alpha}^{-1}-\overline{\hat{\varsigma}}_{k}\right)\right\rangle \mathbf{B}^{-1} \overline{\mathbf{B}}_{\mathbf{k}} \overline{\mathbf{A}}^{\mathbf{T}}\right\} \hat{\mathbf{p}} \\
& \phi=\frac{1}{\pi} \operatorname{Im}\left\{\mathbf{B} \ln \left(\varsigma_{\alpha}-\hat{\varsigma}_{\alpha}\right) \mathbf{A}^{\mathbf{T}}+\sum_{k=1}^{3} \mathbf{B}\left\langle\ln \left(\varsigma_{\alpha}^{-1}-\overline{\hat{\varsigma}}_{k}\right)\right\rangle \mathbf{B}^{-1} \overline{\mathbf{B}} \mathbf{I}_{\mathbf{k}} \overline{\mathbf{A}}^{\mathbf{T}}\right\} \hat{\mathbf{p}}
\end{aligned}
$$

in which the unknown complex function vector $f(z)$ is better to be expressed in terms of the arguments $\zeta_{\alpha}$

$$
\varsigma_{\alpha}=\frac{z_{\alpha}+\sqrt{z_{\alpha}^{2}-a^{2}-p_{\alpha}^{2} b^{2}}}{a-i p_{\alpha} b}, \hat{\varsigma}_{\alpha}=\frac{\hat{z}_{\alpha}+\sqrt{\hat{z}_{\alpha}^{2}-a^{2}-p_{\alpha}^{2} b^{2}}}{a-i p_{\alpha} b}
$$

where

$$
z_{\alpha}=x_{1}+p_{\alpha} x_{2}, \quad \hat{z}_{\alpha}=\hat{x}_{1}+p_{\alpha} \hat{x}_{2}
$$

and $2 \mathrm{a}$ and $2 \mathrm{~b}$ are the length of the major and minor axis of the elliptical hole. The corresponding stress components can be expressed as

$$
\begin{aligned}
\sigma_{i 1}^{*} & =-\frac{1}{\pi} \operatorname{Im}\left\{\mathbf{B}\left\langle\frac{\lambda p_{\alpha}}{\chi}\right\rangle \mathbf{A}^{\mathbf{T}}\right\} \hat{\mathbf{p}}-\frac{1}{\pi} \sum_{k=1}^{3} \operatorname{Im}\left\{\mathbf{B}\left\langle\frac{-2 \varsigma_{\alpha} p_{\alpha}}{\left(1-\varsigma_{\alpha} \overline{\hat{\zeta}}_{k}\right) \chi}\right\rangle \mathbf{B}^{-1} \overline{\mathbf{B}}_{\mathbf{k}} \overline{\mathbf{A}}^{\mathbf{T}}\right\} \hat{\mathbf{p}} \\
\sigma_{i 2}^{*} & =\frac{1}{\pi} \operatorname{Im}\left\{\mathbf{B}\left\langle\frac{\lambda}{\chi}\right\rangle \mathbf{A}^{\mathbf{T}}\right\} \hat{\mathbf{p}}+\frac{1}{\pi} \sum_{k=1}^{3} \operatorname{Im}\left\{\mathbf{B}\left\langle\frac{-2 \varsigma_{\alpha}}{\left(1-\varsigma_{\alpha} \overline{\hat{\varsigma}}_{k}\right) \chi}\right\rangle \mathbf{B}^{-1} \overline{\mathbf{B}}_{\mathbf{k}} \overline{\mathbf{A}}^{\mathbf{T}}\right\} \hat{\mathbf{p}}
\end{aligned}
$$

where $\lambda=2 \varsigma_{\alpha}^{2} /\left(\varsigma_{\alpha}-\hat{\varsigma}_{\alpha}\right), \chi=\left(a-i p_{\alpha} b\right) \varsigma_{\alpha}^{2}-\left(a+i p_{\alpha} b\right)$. The fundamental solutions for an infinite anisotropic medium with a crack of length 2 a can be obtained easily by letting $b=0$ in Eq. (15). 


\section{HFS-FEM formulations}

\subsection{Assumed fields}

To solve the anisotropic problem governed by Eqs. (4)-(6) using HFS-FEM approach, the solution domain $\Omega$ is divided into a series of elements as was done in conventional FEM. For each element, two independent fields, i.e. intraelement field and frame field, are assumed [18]. In this approach, the intraelement displacement fields for a particular element $e$ is approximated in terms of a linear combination of fundamental solutions of the problem as

$$
\mathbf{u}(\mathbf{x})=\left\{u_{1}(\mathbf{x}) \quad u_{2}(\mathbf{x}) \quad u_{3}(\mathbf{x})\right\}^{T}=\mathbf{N}_{\mathbf{e}} \mathbf{c}_{\mathbf{e}} \quad\left(\mathbf{x} \in \Omega_{e}, \mathbf{y}_{s j} \notin \Omega_{e}\right)
$$

where the matrix $\mathbf{N}_{\mathbf{e}}$ and unknown vector $\mathbf{c}_{\mathbf{e}}$ can be further written as

$$
\mathbf{N}_{\mathbf{e}}=\left[\begin{array}{llllll}
u_{11}^{*}\left(\mathbf{x}, \mathbf{y}_{s 1}\right) & u_{12}^{*}\left(\mathbf{x}, \mathbf{y}_{s 1}\right) & \cdots & u_{11}^{*}\left(\mathbf{x}, \mathbf{y}_{s n_{s}}\right) & u_{12}^{*}\left(\mathbf{x}, \mathbf{y}_{s n_{s}}\right) & u_{13}^{*}\left(\mathbf{x}, \mathbf{y}_{s n_{s}}\right) \\
u_{12}^{*}\left(\mathbf{x}, \mathbf{y}_{s 1}\right) & u_{22}^{*}\left(\mathbf{x}, \mathbf{y}_{s 1}\right) & \cdots & u_{12}^{*}\left(\mathbf{x}, \mathbf{y}_{s n_{s}}\right) & u_{22}^{*}\left(\mathbf{x}, \mathbf{y}_{s n_{s}}\right) & u_{23}^{*}\left(\mathbf{x}, \mathbf{y}_{s n_{s}}\right) \\
u_{13}^{*}\left(\mathbf{x}, \mathbf{y}_{s 1}\right) & u_{32}^{*}\left(\mathbf{x}, \mathbf{y}_{s 1}\right) & \cdots & u_{13}^{*}\left(\mathbf{x}, \mathbf{y}_{s n_{s}}\right) & u_{32}^{*}\left(\mathbf{x}, \mathbf{y}_{s n_{s}}\right) & u_{33}^{*}\left(\mathbf{x}, \mathbf{y}_{s n_{s}}\right)
\end{array}\right]
$$

in which $n_{s}$ is the number of source points, $\mathbf{x}$ and $\mathbf{y}_{s j}$ are respectively the field point and source point in the local coordinate system $\left(\mathrm{X}_{1}, \mathrm{X}_{2}\right)$. The components $u_{i j}^{*}\left(\mathbf{x}, \mathbf{y}_{s j}\right)$ is the fundamental solution, i.e. induced displacement component in $i$ direction at the field point $\mathbf{x}$ due to a unit point load applied in $j$-direction at the source point $\mathbf{y}_{s j}$ placed outside the element, which are given by Eq. (13) for general elements or Eq. (15) for special elements with an elliptical hole or crack. In our analysis, the number of source points is taken to be the same as the number of element nodes, which is free of spurious energy modes and can keep the stiffness equations in full rank, as indicated in [16]. The source point $\mathbf{y}_{s j}\left(j=1,2, \ldots, n_{s}\right)$ can be generated by [18]

$$
\mathbf{y}_{s}=\mathbf{x}_{0}+\gamma\left(\mathbf{x}_{0}-\mathbf{x}_{c}\right)
$$

where $\gamma$ is a dimensionless coefficient, $\mathbf{x}_{0}$ is the point on the element boundary (the nodal point in this work) and $\mathbf{x}_{c}$ the geometrical centroid of the element (see Figure 2). Determination of $\gamma$ was discussed in [19] and $\gamma=8$ is used in the following analysis.

The corresponding stress fields can be expressed as

$$
\boldsymbol{\sigma}(\mathbf{x})=\left[\begin{array}{lllll}
\sigma_{11} & \sigma_{22} & \sigma_{23} & \sigma_{31} & \sigma_{12}
\end{array}\right]^{T}=\mathbf{T}_{\mathbf{e}} \mathbf{c}_{\mathbf{e}}
$$

where $\mathbf{T}_{\mathbf{e}}$ is a coefficient matrix with its components $\sigma_{i j k}^{*}(\mathbf{x}, \mathbf{y})$ given by Eq. (14) or Eq. (18) when $\hat{\mathbf{p}}_{i}$ is selected to be $(1,0,0)^{T},(0,1,0)^{T}$ and $(0,0,1)^{T}$, respectively. As a consequence, the traction can be written as

$$
\left\{\begin{array}{lll}
t_{1} & t_{2} & t_{3}
\end{array}\right\}^{T}=\mathbf{n} \sigma=\mathbf{n} \mathbf{T}_{\mathbf{e}} \mathbf{c}_{\mathbf{e}}=\mathbf{Q}_{\mathbf{e}} \mathbf{c}_{\mathbf{e}}
$$



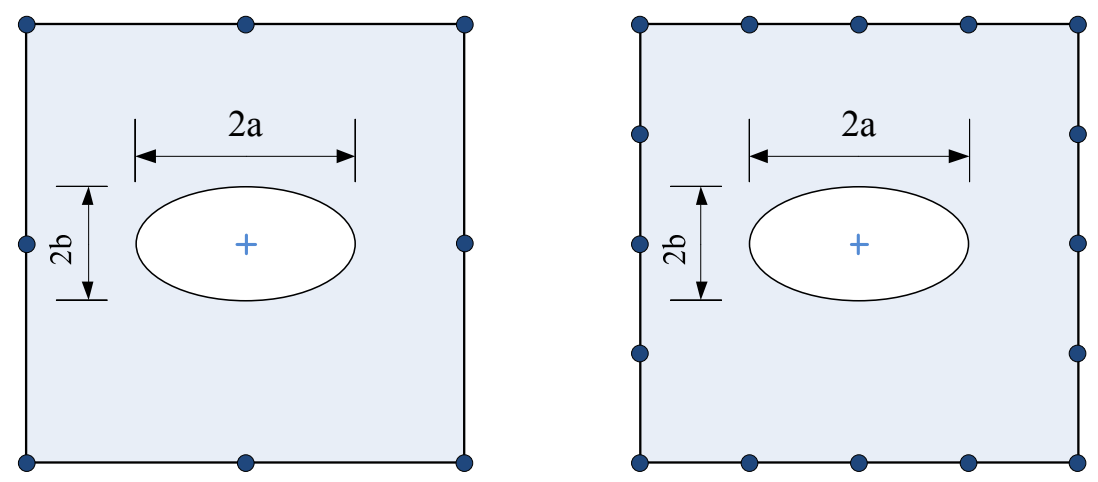

Figure 2: $\quad$ Special elements for elliptical hole or crack.

The unknown $\mathbf{c}_{e}$ in Eq. (19) and Eq. (23) may be calculated using a hybrid technique, in which the elements are linked through an auxiliary conforming displacement frame which has the same form as in conventional FEM (see Fig. 2). Thus, the frame is defined as

$$
\tilde{\mathbf{u}}(\mathbf{x})=\left\{\begin{array}{l}
\tilde{u}_{1} \\
\tilde{u}_{2} \\
\tilde{u}_{3}
\end{array}\right\}=\left\{\begin{array}{l}
\tilde{\mathbf{N}}_{1} \\
\tilde{\mathbf{N}}_{2} \\
\tilde{\mathbf{N}}_{3}
\end{array}\right\} \mathbf{d}_{e}=\tilde{\mathbf{N}}_{e} \mathbf{d}_{e}, \quad\left(\mathbf{x} \in \Gamma_{e}\right)
$$

where the symbol " $\sim$ " is used to specify that the field is defined on the element boundary only, $\tilde{\mathbf{N}}_{e}$ is the matrix of shape functions, $\mathbf{d}_{e}$ is the nodal displacements of elements. Taking the side 3-4-5 of a particular 8-node quadrilateral element (see Figure 2) as an example, $\tilde{\mathbf{N}}_{e}$ and $\mathbf{d}_{e}$ can be expressed as

$$
\begin{gathered}
\tilde{\mathbf{N}}_{e}=\left[\begin{array}{lllllllll}
\mathbf{0} & \mathbf{0} & \overline{\mathbf{N}}_{1} & \overline{\mathbf{N}}_{2} & \overline{\mathbf{N}}_{3} & \mathbf{0} & \mathbf{0} & \mathbf{0}
\end{array}\right] \\
\mathbf{d}_{e}=\left[\begin{array}{llllllllll}
u_{11} & u_{21} & u_{31} & u_{12} & u_{22} & u_{32} & \cdots & u_{18} & u_{28} & u_{38}
\end{array}\right]^{T}
\end{gathered}
$$

where the shape functions are expressed as

$$
\overline{\mathbf{N}}_{\mathbf{i}}=\left[\begin{array}{ccc}
\tilde{N}_{i} & 0 & 0 \\
0 & \tilde{N}_{i} & 0 \\
0 & 0 & \tilde{N}_{i}
\end{array}\right], \mathbf{0}=\left[\begin{array}{ccc}
0 & 0 & 0 \\
0 & 0 & 0 \\
0 & 0 & 0
\end{array}\right]
$$

and $\tilde{N}_{1}, \tilde{N}_{2}$ and $\tilde{N}_{3}$ are expressed by natural coordinate $\xi \in[-1,1]$

$$
\tilde{N}_{1}=-\frac{\xi(1-\xi)}{2}, \tilde{N}_{2}=1-\xi^{2}, \tilde{N}_{3}=\frac{\xi(1+\xi)}{2} \quad(\xi \in[-1,1])
$$




\subsection{Modified functional and element Stiffness matrix for HFS-FEM}

With the assumption of two distinct intra-element field and frame field for elements, we can establish the modified variational principle based on Eqs. (4)(6) for the hybrid finite element method of anisotropic materials [16]. In the absence of the body forces, the hybrid functional $\Pi_{m e}$ for a particular element $e$ is constructed as

$$
\Pi_{m e}=\frac{1}{2} \iint_{\Omega_{e}} \sigma_{i j} \varepsilon_{i j} d \Omega-\int_{\Gamma_{t}} \bar{t}_{i} \tilde{u}_{i} d \Gamma+\int_{\Gamma_{e}} t_{i}\left(\tilde{u}_{i}-u_{i}\right) d \Gamma
$$

where the boundary $\Gamma_{e}$ of the element $e$ is

$$
\Gamma_{e}=\Gamma_{e u} \cup \Gamma_{e t} \cup \Gamma_{e I} \text { and } \Gamma_{e u}=\Gamma_{e} \cap \Gamma_{u}, \Gamma_{e t}=\Gamma_{e} \cap \Gamma_{t}
$$

and $\Gamma_{e I}$ is the inter-element boundary of element $e$. Compared to the functional employed in the conventional FEM, the present hybrid functional is constructed by adding two integral terms related to the intra-element and element frame fields to guarantee the satisfaction of displacement continuity condition on the common boundary of two adjacent elements. To this end, performing a variation of $\Pi_{m}$, one obtains

$$
\delta \Pi_{m e}=\iint_{\Omega_{e}} \sigma_{i j} \delta u_{i, j} d \Omega-\int_{\Gamma_{e t}} \bar{t}_{i} \delta \tilde{u}_{i} d \Gamma+\int_{\Gamma_{e}}\left[\left(\tilde{u}_{i}-u_{i}\right) \delta t_{i}+t_{i}\left(\delta \tilde{u}_{i}-\delta u_{i}\right)\right] d \Gamma
$$

Applying Gaussian theorem, it can be demonstrated that the Euler equations for Eq. (32) result in Eqs. (4)-(6). In addition, the existence of extremum of functional (30) can be easily proofed by "second variational approach" as well. Therefore, we can conclude that functional (30) can be used for deriving hybrid finite element formulations.

Using Gaussian theorem and equilibrium equations, the functional in Eq. (30) can be simplified to final expression for the HT finite element model

$$
\Pi_{m e}=-\frac{1}{2} \int_{\Gamma_{e}} t_{i} u_{i} d \Gamma+\int_{\Gamma_{e}} t_{i} \tilde{u}_{i} d \Gamma-\int_{\Gamma_{t}} \bar{t}_{i} \tilde{u}_{i} d \Gamma
$$

Substituting Eqs. (19), (24) and (25) into the above functional (33) yields the formulation as

$$
\Pi_{m e}=-\frac{1}{2} \mathbf{c}_{e}{ }^{T} \mathbf{H}_{e} \mathbf{c}_{e}+\mathbf{c}_{e}{ }^{T} \mathbf{G}_{e} \mathbf{d}_{e}-\mathbf{d}_{e}{ }^{T} \mathbf{g}_{e}
$$

where

$$
\mathbf{H}_{e}=\int_{\Gamma_{e}} \mathbf{Q}_{e}^{\mathrm{T}} \mathbf{N}_{e} \mathrm{~d} \Gamma, \quad \mathbf{G}_{e}=\int_{\Gamma_{e}} \mathbf{Q}_{e}^{\mathrm{T}} \tilde{\mathbf{N}}_{e} \mathrm{~d} \Gamma, \quad \mathbf{g}_{e}=\int_{\Gamma_{t}} \tilde{\mathbf{N}}_{e}^{\mathrm{T}} \overline{\mathbf{t}} d \Gamma
$$

To enforce inter-element continuity on the common element boundary, the unknown vector $\mathbf{c}_{\mathrm{e}}$ should be expressed in terms of nodal degrees of freedom $\mathbf{d}_{\mathrm{e}}$. The stationary condition of the functional $\Pi_{m e}$ with respect to $\mathbf{c}_{\mathrm{e}}$ and $\mathbf{d}_{\mathrm{e}}$, respectively, yields 


$$
\frac{\partial \Pi_{m e}}{\partial \mathbf{c}_{e}{ }^{\mathrm{T}}}=-\mathbf{H}_{e} \mathbf{c}_{e}+\mathbf{G}_{e} \mathbf{d}_{e}=\mathbf{0}, \quad \frac{\partial \Pi_{m e}}{\partial \mathbf{d}_{e}{ }^{\mathrm{T}}}=\mathbf{G}_{e}{ }^{T} \mathbf{c}_{e}-\mathbf{g}_{e}=\mathbf{0}
$$

from which the relationship between $\mathbf{c}_{\mathrm{e}}$ and $\mathbf{d}_{\mathrm{e}}$, and the stiffness equation can be obtained as

$$
\mathbf{c}_{e}=\mathbf{H}_{e}^{-1} \mathbf{G}_{e} \mathbf{d}_{e}, \quad \mathbf{K}_{e} \mathbf{d}_{e}=\mathbf{g}_{e}
$$

where $\mathbf{K}_{e}=\mathbf{G}_{e}{ }^{\mathrm{T}} \mathbf{H}_{e}{ }^{-1} \mathbf{G}_{e}$ is the element stiffness matrix with symmetric properties. The numerical calculations for $\mathbf{H}_{e}, \mathbf{G}_{e}$ and $\mathbf{g}_{e}$ can resort to the popular Gauss integration as used in FEM and BEM.

\section{Numerical examples}

In this section, two examples are presented to assess the accuracy and efficiency of the present method. In the examples shown below, $L$ and $W$ stand for the length and width of the plate, respectively; $2 a$ and $2 b$ are the lengths of the major and minor axis of the ellipse. In the computation, the infinite plate with an elliptical hole is modeled by a large square plate with $L / W=1$ and $W / \mathrm{a}=100$ [20].

\subsection{An anisotropic plate with an elliptic hole}

As shown in Figure 3, an infinite plate containing an elliptical hole is investigated in this example. A uniform tension of $\sigma_{0}=1 \mathrm{GPa}$ is applied in $x_{2}$ direction. The material parameters of the orthotropic plate are taken as $\mathrm{E}_{\mathrm{l}}=11.8 \mathrm{GPa}, \mathrm{E}_{2}=5.9 \mathrm{GPa}, \mathrm{G}_{12}=0.69 \mathrm{GPa}, \mathrm{v}_{12}=0.071$ [12]. In this analysis, it is not necessary to use lots of elements to capture the concentrated stress as done in the traditional FEM. A relatively coarse mesh can be employed and the elliptical hole can be considered by only one special element. The mesh configuration
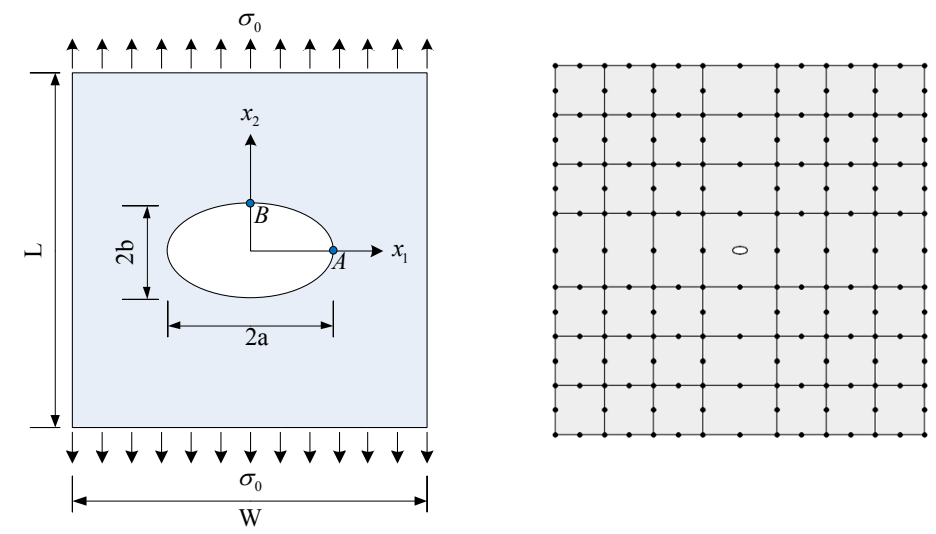

Figure 3: An orthotropic plate with an elliptic hole under tension and its mesh configuration. 
with one 8-node special hybrid element and 48 8-node hybrid elements with a total of 176 nodes is shown in Figure 3. The effect of the locations of source points on the convergence and accuracy of the stress and displacements have been investigated in our previous work [18], which will be omitted in this paper. Figure 4 shows the variations of the stress concentration factors (SCF) of the plate with the increasing ratio $\mathrm{b} / \mathrm{a}$ of the ellipse. It can be seen from Figure 4 that the SCF of the plate decreases along with an increase in the ratio b/a and the results calculated by HFS-FEM have a very good agreement with the BEM solutions [12].

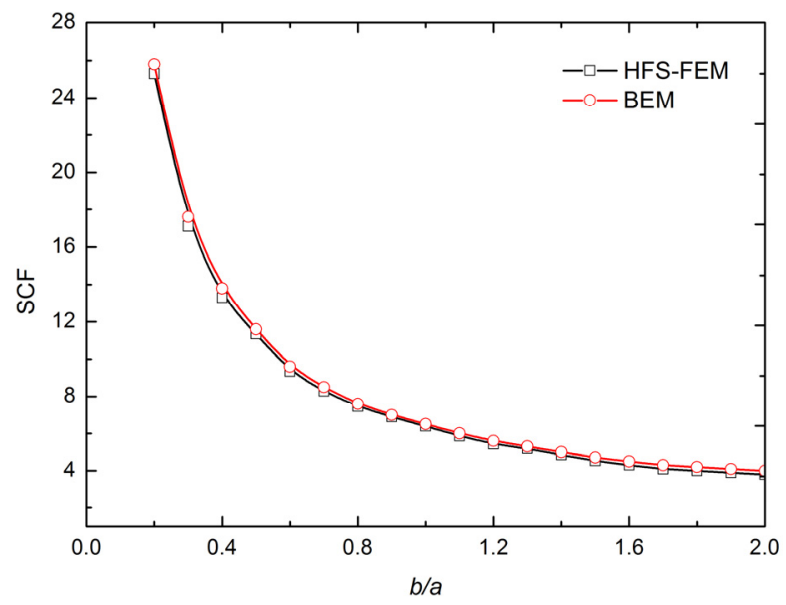

Figure 4: Variations of SCF with the ratio b/a of the elliptical hole.

\subsection{An anisotropic plate with a centre crack}

The extreme case of an elliptical hole (i.e. crack) is considered in this example. By letting the minor axis b approach to zero, an elliptical hole can be made into a crack of length $2 \mathrm{a}$. The geometry and loading for this problem are the same as that in Figure 3 with $\mathrm{b}=0$ and $\mathrm{L} / \mathrm{W}=1$. The plate is subjected to a uniform tensile stress $\sigma_{0}=1 \mathrm{GPa}$ in the $\mathrm{x}_{2}$-direction. The plate is composed of the orthotropic materials whose mechanical properties are $\mathrm{E}_{1}=114.8 \mathrm{Gpa}, \mathrm{E}_{2}=\mathrm{E}_{3}=$ $11.72 \mathrm{Gpa}, \mathrm{G}_{12}=\mathrm{G}_{13}=\mathrm{G}_{23}=9.65 \mathrm{Gpa}, \mathrm{v}_{12}=\mathrm{v}_{13}=\mathrm{v}_{23}=0.21$. The variation of the SIF $\mathrm{K}_{\mathrm{I}}$ with respect to the crack length $a$ is shown in Figure 5. It can be seen that the calculated $\mathrm{K}_{\mathrm{I}}$ by HFS-FEM also agree very well with those from Snyder and Cruse [21]. It can be concluded that the new HFS-FEM method is able to treat these two kinds of defects (elliptical holes and cracks) in a uniform scheme for facilitating the modelling of anisotropic materials with defects.

\section{Conclusions}

In this work a new hybrid finite element formulation (HFS-FEM) with special elements for elliptic hole/crack has been developed to provide an efficient 


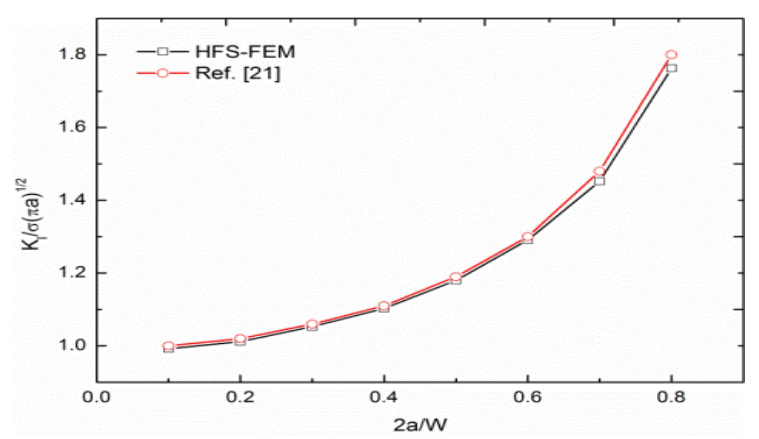

Figure 5: $\quad$ The variation of $\mathrm{K}_{\mathrm{I}}$ with the increasing crack length $2 \mathrm{a}$.

approach for stress analysis of anisotropic materials with defects. Since the special fundamental solutions used in our calculation have exactly satisfied the boundary conditions for the hole/crack as a priori, we can use only one special element to model the hole/crack region and there is no need to discretize the hole/crack boundary. This feature leads to a vast amount of mesh reduction and computational effort saving. The numerical examples for anisotropic composite plates with an elliptic hole or a crack were employed to assess the performance of the proposed method. Numerical results show that the present method can effectively capture the stress field around the elliptical holes/cracks by using much less meshes. It can be concluded that the present approach can be employed to effectively calculate the SCFs or SIFs in anisotropic materials with holes or cracks.

\section{References}

[1] Kushch, V.I., Sevostianov I. and Mishnaevsky, L., Stress concentration and effective stiffness of aligned fiber reinforced composite with anisotropic constituents. International Journal of Solids and Structures, 45(18-19), pp. 5103-5017, 2008.

[2] Qin, Q.H., Mai, Y.W. and Yu, S.W., Some problems in plane thermopiezoelectric materials with holes. International Journal of Solids and Structures, 36(3), pp. 427-39, 1999.

[3] Tu, C.H., Chen, C.S. and Yu, T,T., Fracture mechanics analysis of multiple cracks in anisotropic media. International Journal for Numerical and Analytical Methods in Geomechanics, 35(11), pp. 1226-42, 2011.

[4] Yu, S.W. and Qin, Q.H., Damage analysis of thermopiezoelectric properties: part II-effective crack model, Theoretical and Applied Fracture Mechanics, 25, 279-288, 1996.

[5] Qin, Q.H. and Mai, Y.W., Crack growth prediction of an inclined crack in a half-plane thermopiezoelectric solid. Theoretical and Applied Fracture Mechanics, 26(3), pp. 185-91, 1997.

[6] Lekhnitskii, S.G., Theory of elasticity of an anisotropic body, Mir Publishers: Moscow, 1981. 
[7] Ting, T.C.T., Anisotropic elasticity: Theory and applications. Oxford Science Publishers: New York, 1996.

[8] Qin, Q.H., General solutions for thermopiezoelectrics with various holes under thermal loading. International Journal of Solids and Structures, 37(39), pp. 5561-5578, 2000.

[9] Qin, Q.H. and Mai, Y.W., BEM for crack-hole problems in thermopiezoelectric materials. Engineering Fracture Mechanics, 69(5), pp. 577-588, 2002.

[10] Dhanasekar, M., Han, J. and Qin, Q.H., A hybrid-Trefftz element containing an elliptic hole. Finite Elements in Analysis and Design, 42, pp. 1314-1323, 2006.

[11] Yu, P.S., Guo, W.L., She, C.M., Zhao, J.H., The influence of Poisson's ratio on thickness-dependent stress concentration at elliptic holes in elastic plates. International Journal of Fatigue, 30(1), pp. 165-171, 2008.

[12] Hwu, C.B., Yen, W.J., Greens-functions of 2-dimensional anisotropic plates containing an elliptic hole. International Journal of Solids and Structures, 27(13), pp. 1705-1719, 1991.

[13] Cao, C., Qin, Q.H. and Yu, A., A new hybrid finite element approach for three-dimensional elastic problems. Archives of Mechanics, 64(3), pp. 261-292, 2012.

[14] Cao, C., Yu, A. and Qin, Q.H., Evaluation of Effective Thermal Conductivity of Fiber-Reinforced Composites. International Journal of Architecture, Engineering and Construction, 1(1), pp. 14-29, 2012.

[15] Cao, C., Qin, Q.H. and Yu, A., Hybrid fundamental-solution-based FEM for piezoelectric materials. Computational Mechanics, 50(4), pp. 397-412. 2012.

[16] Qin, Q.H., The Trefftz Finite and Boundary Element Method. WIT Press: Southampton, 2000.

[17] Qin, Q.H., Thermoelectroelastic Green's function for a piezoelectric plate containing an elliptic hole. Mechanics of Materials, 30(1), pp. 21-29, 1998.

[18] Wang, H. and Qin, Q.H., Fundamental-solution-based finite element model for plane orthotropic elastic bodies. European Journal of Mechanics-A/Solids, 29(5), 801-09, 2010.

[19] Wang, H., Qin, Q.H. and Kang, Y.L., A new meshless method for steadystate heat conduction problems in anisotropic and inhomogeneous media. Archive of Applied Mechanics, 74(8): 563-79, 2005.

[20] Piltner, R., Some remarks on finite elements with an elliptic hole. Finite Elements in Analysis and Design, 44(12-13), 767-72, 2008.

[21] Snyder, M.D. and Cruse, T.A., Boundary-integral equation analysis of cracked anisotropic plates. International Journal of Fracture, 11, pp. 315$328,1975$. 